\title{
Bushfire Emergency Response Simulation
}

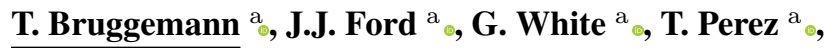 \\ ${ }^{a}$ Science and Engineering Faculty, Queensland University of Technology, Australia \\ Email: t.bruggemann@qut.edu.au
}

\begin{abstract}
Improving the ability to model, manage and communicate the impact of uncertainty in complex interacting systems is becoming increasingly important. This is particularly important for defence as the nature of warfare is rapidly changing due to the incursion of novel defence technologies. The dominant attribute of modern warfare is its network-centric characteristic, which can be both an enabling and a limiting factor. Network-centric warfare refers to a potentially large number of entities or assets with varying capabilities for autonomous decision making whose coordinated fighting power is enabled through information exchange within a constrained system structure that can be described in terms of interacting networks. Such networks are known to be able to exhibit complex behaviours such as rapid phase transitions, oscillations, chaos, disturbance rejection, and adaptation. Understanding the potential for complex behaviours associated with particular military operations is essential to establish appropriate simulation and data collection processes that inform decisions about force design and asset acquisition. To explore these concepts in an unclassified manner, this project developed a bushfire emergency response simulator as a surrogate for similar Defence problems.
\end{abstract}

This paper describes our multi-agent simulation design which is intended to capture key dynamic characteristics of emergency response operations including individual fire response asset behaviours and team hierarchies in perception and decision making. This new bushfire emergency response simulator includes assets to be protected, command and control assets, fixed and mobile sensing assets, and assets within the response team with perception, decision, action and communication capabilities and with different capabilities to move and fight the fire. A new cellular automata type bushfire model with dynamical cell interactions considering fuel and wind is introduced, that enables simulation of a greater range of fire behaviours with higher fidelity, than simple cellular automata models.

This is the second in a series of papers describing bushfire emergency response simulation as a surrogate for Defence problems to explore the quantification of uncertainty in modelling, simulation and analysis of complex systems. This paper describes in further detail the bushfire emergency response simulator which was applied to uncertainty quantification in Bruggemann et al. (2019).

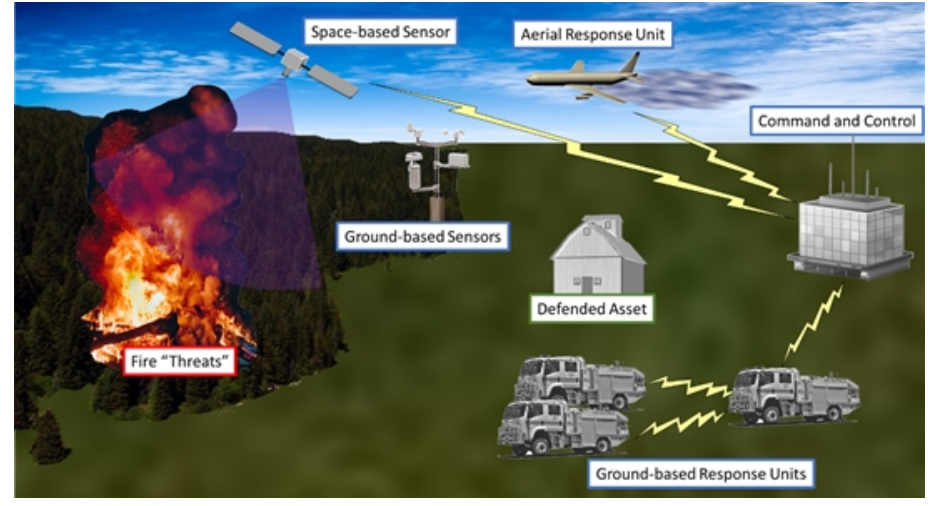

Figure 1. Simplified operational context diagram of bushfire emergency response.

Keywords: Fire spread model, multi-agent simulation, cellular automata, Monte Carlo, bushfire emergency response 


\section{INTRODUCTION}

Understanding complex interacting systems by quantifying uncertainty in different situations and analyzing how uncertainties interact is becoming an increasingly important part of making decisions about developing and shaping future defence capability. New simulation tools are needed to model, manage and communicate the impact of uncertainty in complex dynamical systems modelling, simulations and analysis. A bushfire emergency response simulator is proposed and developed in this project as an unclassified surrogate for similar defence problems.

An early simulator of bushfires and fire fighting was the Fire Chief microworld generating program for studying human decision making (Omodei and Wearing (1995)). RescueModel was the first multi-agent simulation for bushfire emergency response and was adapted from a military operations simulator (Au et al. (2000)). It incorporates the environment, fire spread, and agent-based tactics and decisions and has been used for analyzing agent architectures including access to the environment, team work and command and control.

In past years numerous tools have been developed using agent-based models and multi-agent systems for planning and tactical decision making in evacuation and disaster or emergency response (Hu et al. (2012); HoseinDoost et al. (2019); Shahparvari et al. (2016); Singh and Padgham (2017); Hawe et al. (2012); Gonzalez (2009); Padgham et al. (2014); Intini et al. (2019); Massaguer et al. (2006)). These also include decision support systems for bush fire fighting and prevention, which combines environmental simulation with multiagent systems to present results of different decisions for decision-maker evaluation (Engelmann and Fiedrich (2007); Jaber et al. (2001)). In addition to emergency response support tools there exists bushfire spread simulators used specifically to aid in the prediction and management of bushfires such as PHOENIX Rapidfire (Tolhurst et al. (2008)), AUSTRALIS (Johnston et al. (2008)), and SPARK (Miller et al. (2015)). Bushfire spread simulators can be used to model a bushfire's spatial and temporal characteristics in different scenarios including and model the environment, fire behaviour, wind, terrain, fuel, and asset impact models enabling predictions, statistics and visualization of bushfire spread to aid decision makers (Miller et al. (2015); Sullivan (2009)).

Since RescueModel, there are few simulation tools for bushfire emergency response that captures dynamical interactions between a fire fighting team, support assets and a bushfire threat. In contrast to RescueModel, our simulator is intended for addressing uncertainty and complexity (see Bruggemann et al. (2019) for an example of the simulator applied to uncertainty quantification). As part of the emergency response simulation, we present a fire spread model based on a grid cellular automata (CA) approach which is simpler and computationally faster than continuous models (Hilton et al. (2018)), since it is intended to characterize the global behaviour of bushfire threats in response to fuel and wind stimulus, over a wide area. In CA for fire spread models, cell states are typically discrete and cell to cell dynamics are governed by simple deterministic or probabilistic rules (Alexandridis et al. (2008); Yongzhong et al. (2005); Liu et al. (2018)). By contrast, in our approach the cell states are bounded continuous and the cell behaviours and interactions are governed by a differential equation and its parameters. We propose the logistic function as a way to model the continuous change in temperature and fuel in each cell, that allows simulation of a greater range of fire threat behaviours with higher fidelity than simple models. The fire model may be tuned to match experimental data via the model parameters. Cell to cell dynamics are deterministic but stochasticity can be introduced via stochastic inputs such as the wind.

\section{MISSION AND OPERATIONAL ENVIRONMENT DEFINITION}

Fig. 1 presents a simplified operational context diagram of bushfire emergency response. We consider the mission to include a Bushfire Response Team (BRT) fighting a Bushfire Threat (BFT): Spot bushfires can occur at any location within the considered area of operations. The area is characterised by its fuel distribution and topography. The fire is also affected by wind direction and speed; Defended Assets (DAs) are ground-based assets that are vulnerable to the BFT. They may or may not have fire-fighting capabilities. Ground-based Sensing Units (GSUs) are sensors are spatially distributed over the area of operations, They provide local high-resolution information related to the state of the BFT and wind. Space-based Sensing Units (SSUs) are satellite-like sensors that can provide global information related to the state of the BFT and response team at specific times. Namely, they may provide a service under a limited availability, at specific times, and maybe limited by atmospheric conditions. Global Command and Control Units (GC2) are global command and control (C2) units that set the BFT response strategy and provides execution plans for the different response teams and units. Aerial-response Units (ARUs) have sensing capability as well as the capability to extinguish the fire. 
These units provide localised high-power fire-fighting capability. These units communicate with the GC2 unit. Local Response C2 Units (LC2s) are mobile local C2 units that communicate with their associated fire-fighting response units and also the global $\mathrm{C} 2$ unit. These units set the BFT strategy and provide execution plans for their assigned ground-based response units at the local level. Ground-based Response Units (GRUs) are mobile units with local fire-sensing as well as local low-power fire-fighting capabilities. GRUs can communicate with LC2 to which they are assigned and have local decision making capabilities.

\subsection{Environment and Threat Model}

The threat environment is modelled as a two-dimensional spatial grid of cells defined by rows and columns $X=\left\{1,2, \ldots, x_{\max }\right\}$ and $Y=\left\{1,2, \ldots, y_{\max }\right\}$, and cells are identified by their cell coordinates $i \in X$, $j \in Y$. The bushfire threat is modelled as cells each having a temperature $T$, fuel level $F$ and wind vector $w_{v}$ state.To each cell $i j$ is associated the dynamical state-space model:

$$
\begin{aligned}
& \dot{T}_{i j}= \begin{cases}\theta_{1} T_{i j}\left(1-T_{i j}\right)+\sum_{k=1}^{8} \theta_{5}^{-1}\left(u_{i j}^{k}\right)\left(\left(1-S_{w}\right) \vee I_{k}\left(w_{v}\right)\right) & \text { if } F_{i j}>\theta_{2}, W=0 \\
-\theta_{13} T_{i j} & \text { if } W=1 \\
-\theta_{3} T_{i j} & \text { otherwise, }\end{cases} \\
& \dot{F}_{i j}=-\theta_{4} T_{i j} F_{i j},
\end{aligned}
$$

The first term in (1) is an autonomous logistic model which has two equilibria: $\bar{T}_{i j}=0$ and $\bar{T}_{i j}=1$. If the initial temperature is in the range $T_{i j} \in[0,1]$ then the temperature will tend asymptotically to 1 , and $\theta_{1} \in \mathbb{R}_{\geq 0}$ determines the rate of temperature increase, provided that there is enough fuel $\left(F_{i j}>\theta_{2}\right.$ where $\theta_{2} \in[0,1]$ is minimum fuel required to trigger temperature increase) and the cell is not wet (cell wetness $W=0$ ). The second term in (1) is a forcing term which ignites the fire (temporarily transitions the state $T_{i j}$ from 0 to a positive value) depending upon the net temperature of the 8 adjacent cells. $C_{k}$ with $k=\{1,2,3,4,5,6,7,8\}$ are the 8 neighbouring cells of the cell $i, j$, shown in Fig. 2. Ignition from neighbour cells is modelled by $u_{i j}^{1} \triangleq \theta_{7} \mu\left(T_{i+1, j}-\theta_{6}\right), u_{i j}^{2} \triangleq \theta_{7} \mu\left(T_{i-1, j}-\theta_{6}\right)$, $u_{i j}^{3} \triangleq \theta_{7} \mu\left(T_{i, j-1}-\theta_{6}\right), u_{i j}^{4} \triangleq \theta_{7} \mu\left(T_{i, j+1}-\theta_{6}\right), u_{i j}^{5} \triangleq \theta_{7} \mu\left(T_{i+1, j-1}-\theta_{6}\right), u_{i j}^{6} \triangleq \theta_{7} \mu\left(T_{i+1, j+1}-\theta_{6}\right)$, $u_{i j}^{7} \triangleq \theta_{7} \mu\left(T_{i-1, j-1}-\theta_{6}\right), u_{i j}^{8} \triangleq \theta_{7} \mu\left(T_{i-1, j+1}-\theta_{6}\right)$, where $\mu(\cdot)$ is the Heaviside unit-step function, $\theta_{6} \in \mathbb{R}_{\geq 0}$ is the sensitivity of a cell's temperature to its neighbours, $\theta_{7} \in \mathbb{R}_{\geq 0}$ is the amplitude of an ignition pulse. Here $S_{w}$ is binary where $S_{w}=0$ represents the case of no wind present and $S_{w}=1$ represents the case of wind present; and $w_{v}$ is a wind vector with wind speed $r_{w}$ centred at the cell $i, j$ whose direction aligns with the cell's wind direction $\beta$. Then, depending on which cell the vector points to, it can trigger the ignition of a neighbour cell, for example $i+1, j+1$ as shown in Fig. 2. That is, $I_{k}\left(w_{v}\right) \triangleq 1$ if $w_{v}$ points to cell $k \in C_{k}$ and 0 otherwise.

Other parameters for fire behaviour include $\theta_{3}$ which is the rate of temperature decrease if $F \leq \theta_{2}$ and cell wetness $W=0, \theta_{4}$ is the rate of fuel burn, $\theta_{5}$ is the rate of decay of the ignition pulses. A water effectiveness parameter $\theta_{13}$ is the rate of temperature decrease if water is applied. As $T$ goes to 1 , the fuel starts burning and the amount of fuel reduces with an exponential decay with time constant $\theta_{4}^{-1}$. Once reached, the cell will remain at $T=1$ until the fuel is consumed below a threshold $\theta_{2}$. This condition results in a model switch with an exponential decay of the temperature of the cell with time constant $\theta_{3}^{-1}$.

How fire propagates between neighbouring cells is illustrated by Fig. 3 which shows the temperature and fuel curves for two neighbouring cells. Initially, the amount of fuel in each cell is $F=0.7$ and temperature $T=0$. After cell one is ignited to $T=0.3$, the second cell is ignited shortly after and the temperature in both cells increases as the fuel decreases. When the fuel drops below $F=\theta_{2}=0.05$ the temperature decreases. A fire simulation is presented in Fig. 4 which shows the fire temperature at 70 seconds. This simulation was initialized with two spot fires and uniformly distributed fuel.

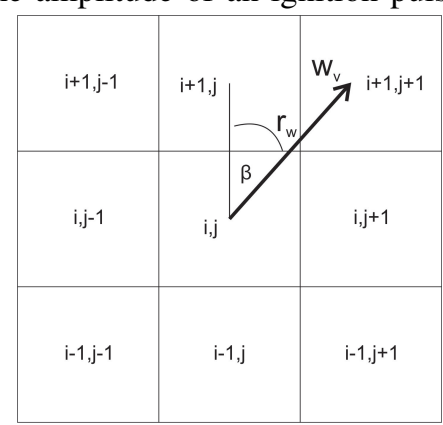

Figure 2. BFT Grid

representation with wind vector

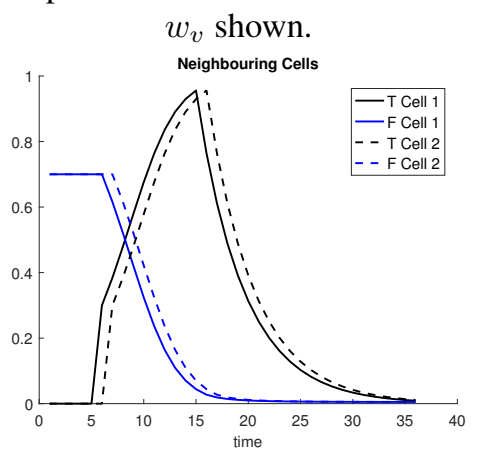

Figure 3. Fuel and Temperature of two neighbouring cells. 


\subsection{Generic Agent Model}

The simulation includes a bush fire threat interacting with a team of assets. The assets are modelled as a set of agents $A$ being independent entities acting under perception $P$, decision $D$ and action $U$ and communication $C$ programs. Four different types of agents are considered including command and control-only agents $A_{g c 2}=\left\{P_{g c 2}, D_{g c 2}, C_{g c 2}\right\}$, fighting response units $A_{g r u}=\left\{P_{g r u}, D_{g r u}, U_{g r u}, C_{g r u}\right\}$, sensing units $A_{g s u}=\left\{P_{g s u}, C_{g s u}\right\}$ and defended assets $A_{d a}$.

The agents are modelled as a simple reflex agents which act only upon the current perception states and follows condition-action rules (Wooldridge (2009)).

Fig. 5 represents GC2, GSU and GRU agents and their interactions with the fire threat and environment and each other. GRU and GSU perceptions of the threat and environment are via direct sensing but GCU perception of the threat and environment is via communication with GRU and GSU. GC2 can command GRU agents which can move in the environment and deploy water on the fire.

States, parameters and models common to all agents are described next, followed by the states, parameters and models unique to each agent type.

States and Parameters: States common to all agents include $\boldsymbol{x}_{\boldsymbol{g e n}}=[a, x, y]$ with availability

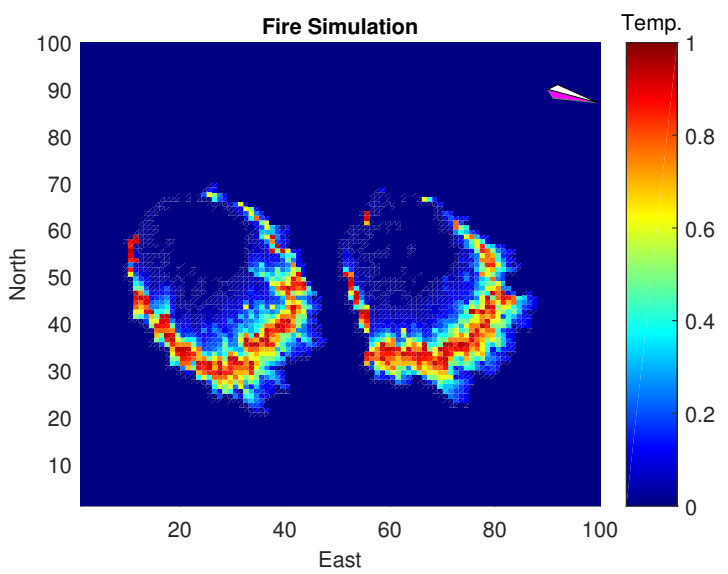

Figure 4. Temperature at 70 seconds with uniform fuel and random wind directions.

$a \in\{0,1\}$ and location $(x, y) \in \mathbb{R}$. A parameter common to all agents is $\theta_{\text {gen }}=\delta_{\text {danger }} \in[0,1]$ which is a temperature threshold above which an asset will be burnt by fire i.e. $a=0$ if $T>\delta_{\text {danger }}$. Additional states and parameters are described in the following models.

Sensor: A threat is defined as a cell having a temperature exceeding a threat threshold $\delta_{\text {threat }} \in[0,1]$. It is convenient to represent all cells as a list $\boldsymbol{T}$ where and $c \in\{1, \ldots, X \times Y\}$ indexes a cell in the list. The sensor model considers detection and measurement of threats within a sensing range $r_{\text {sense }} \in$ $\mathbb{R}_{\geq 0}$ around an agent's location at $(x, y)$. Let $O$ be the set of threats sensed by an agent such that $O=\{c \in$

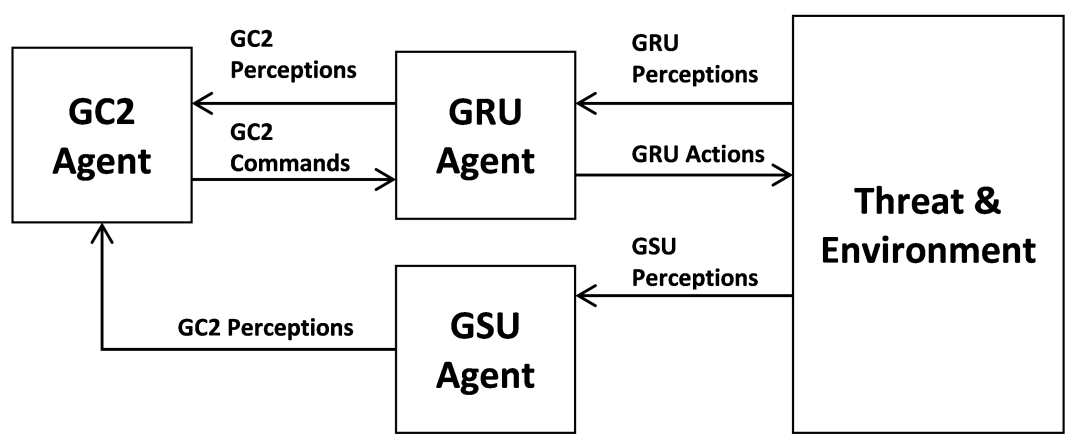

Figure 5. GC2, GSU and GRU agents and their interactions with the fire threat and environment and each other.

$\left.\boldsymbol{T} \mid d(c,(x, y)) \leq r_{\text {sense }}, \hat{T}(c)>\delta_{\text {threat }}\right\}$, where $d(c,(x, y))$ is Euclidean distance between cell $c$ and coordinate $(x, y), \hat{T}=T+v_{t}$ is the cell temperature measurements assuming additive sensor noise $v_{t}$.

Motion: The agent motion in the 2D plane was modelled by a simple car model (LaValle (2006)) with PID feedback-controlled speed $V \in\left[0, V_{\max } \in \mathbb{R}_{\geq 0}\right]$ and heading rate $|\dot{\psi}| \in\left[0, \dot{\psi}_{\max }\right]$ to a movement goal location $(x, y)_{g} \in \mathbb{R} . V_{\max }$ and $\dot{\psi}_{\max }$ are speed and heading-rate constraints.

Communication: An adjacency list $L$ was used to describe the possible interactions between agents, where $j \in L(i)$ if it is possible for agent $i$ to interact with agent $j$, allowing simple modelling of communication channels. Information received from other agents via communication is stored in $C$ for each agent.

\section{3 $A_{\text {gru }}$ Model}

States: States are $\boldsymbol{x}_{\boldsymbol{g r u}}=\left[\boldsymbol{x}_{\boldsymbol{g e n}}, \psi, w, O, I,(x, y)_{g}, c_{g}, c_{d}, C\right]$ where $w \in[0,1]$ is water tank level, $I$ are sensed threat intensities, $c_{g}$ are target cells where water may be deployed, $c_{d}$ are cells to be defended (cells where DA are located). 
Perception: $A_{g r u}$ are equipped with a sensor that measures threats $O$. Threat data may also be received from communication with a different agent $j$ i.e. $C_{i}=\left\{O_{j}\right\}$. An agent's perception from its own sensors and via communication is then $P=f\left(\left\{O_{i}, O_{j}\right\}\right)$. Given that the objective is to protect a DA, we can define a "threat intensity" $I$ as being how close each sensed threat is to a DA, where $I=\left(d\left(c_{d}, c_{g}\right)\right)^{-1}, c_{g} \in\left\{O_{i}, O_{j}\right\}$. Each agent may have a different perception of threat intensities, depending on its location, sensing range and sensing resolution.

Decision and Action: $A_{g r u}$ decision logic activates agent behaviours on the basis of received information or internal states. Decision making includes choosing movement goals $(x, y)_{g}$ and target goals $c_{g} \in O$. For example, to decide to target cells with the highest intensity, the decision rule might be to choose the cell $c_{g} \in O$ with the largest intensity $I$. Decision logic about whether to engage or not is also governed by the amount of water $w$ and GRU availability, and whether or not the target is in range. Four modelled behaviours include (1) Observe - monitor fires by its sensors; (2) Move to engage - move to $(x, y)_{g}$ to engage the fire; (3) Engage - deploy water in a target cell $c_{g}$ when located within water range $r_{w a t e r} \in \mathbb{R}_{\geq 0}$, then $w$ decreases at water consumption rate $W_{\text {loss }}$; (4) Flee - retreat from any immediate danger $T>\delta_{\text {danger }}$ that is within a safety separation radius $r_{\text {safety }} \in \mathbb{R}_{\geq 0}$.

Parameters: Parameters are $\boldsymbol{\theta}_{\text {gru }}=\left[\theta_{\text {gen }}, V_{\text {max }}, \dot{\psi}_{\text {max }}, \delta_{\text {threat }}, r_{\text {sense }}, \delta_{\text {danger }}, r_{\text {safety }}, r_{\text {water }}, W_{\text {loss }}, v_{t}\right]$.

\section{4 $A_{g c 2}$ Model}

States: $\boldsymbol{x}_{\boldsymbol{g c 2}}=\left[\boldsymbol{x}_{\boldsymbol{g e n}}, T O_{1 . . n_{\max }}, C\right]$ where $T O$ are tracked threat objects, $n_{\max }$ is the maximum number of TO possible to be tracked.

Perception: An $A_{g c 2}$ perceives the fire at a global level, as a set of $n$ threat objects $T O_{1 . . n}$ where each $T O$ is a cluster of cells. While response and sensing agents perceive threats at a local level, as individual cells, in essence, the $T O$ can be considered models of perceptive abstractions and are illustrated in Fig. 6. Dependency between high-level $A_{c 2}$ perception and low-level sensing or response unit perceptions enables analysis of complex behaviours and tactical and strategic decision strategies.

Perception is modelled by simulated threat detection, classification and tracking. Threat objects $T O$ are clusters of cells and are constructed by fusing and classifying cell ID and temperature data received by communication from the sensing and response agents. The threat classification is by k-nearest neighbour (Cunningham and Delany (2007)).

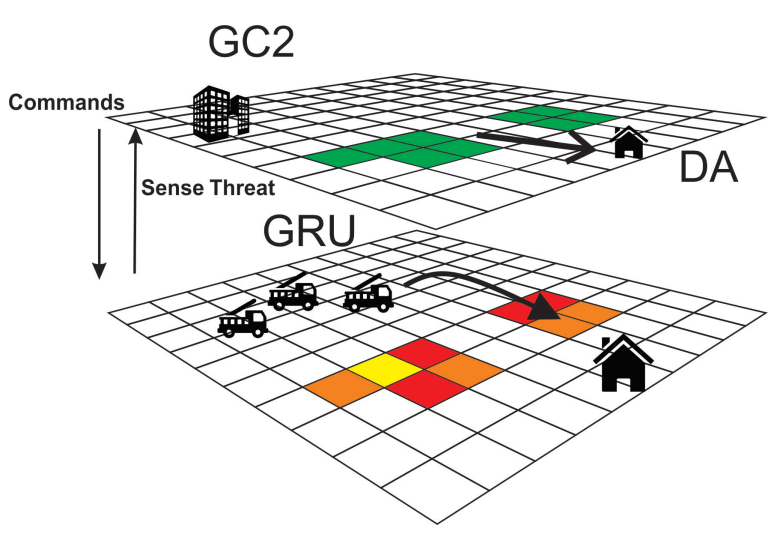

Figure 6. Hierarchical feedback between GC2 and GRU in protection of DA.

The $A_{g c 2}$ then tracks the state of each $T O$ which is $T O=\left[a, x_{c}, y_{c}, \dot{x}_{c}, \dot{y}_{c}, \psi_{c},|T O|,|T O|, \operatorname{mean}\left(T_{k} \in T O\right)\right]$ where $a$ is the availability of the $T O, x_{c}, y_{c}$ is the centroid, $\psi_{c}$ is the heading, $|T O|$ is the size or number of cells of the $T O$, and mean $\left(T_{k} \in T O\right)$ is the average temperature of cells in the TO.

Decision:The GC2 can issue move to or fire at commands to a particular $k \in T O$ which is an individual TO in the set. A C2 strategy is defined as mapping from perceived $T O$ and agent states to a particular $k$. Different task assignment strategies can be programmed such as assign GRU to the $T O$ closest to the DA, or the largest or fastest growing TO. More sophisticated decision models can employ minimum risk strategies. This is outside the scope of this paper.

Parameters: $\boldsymbol{\theta}_{\boldsymbol{g c 2}}=\left[\theta_{\text {gen }}, n_{\max }, r_{\min }, O_{\max }, \delta_{\text {threat }}\right]$, where $n_{\max }$ is the maximum number of $T O$ trackable, $r_{\text {min }}$ the minimum distance between cells to be classified as a new TO, $O_{\max }$ the maximum number of cells in a $T O$ (the maximum $T O$ size).

\section{5 $A_{g s u}, A_{d a}, A_{s s u}$ and $A_{a r u}$ Models}

For perception, $A_{g s u}$ use the sensor and communication models of Section 2.2. The states for $A_{g s u}$ are: $\boldsymbol{x}_{\boldsymbol{g s u}}=\left[\boldsymbol{x}_{\boldsymbol{g e n}}, O_{1 . . n_{\max }}, C\right]$. Parameters are $\boldsymbol{\theta}_{\boldsymbol{g s u}}=\left[\theta_{\text {gen }}, r_{\text {sense }}, V_{t}, \delta_{\text {threat }}\right]$. The $A_{\text {ssu }}$ model can be based on the $A_{g s u}$ model with larger sensing range and noise characteristics to model satellite-based sensing. 
Satellite availability can be modelled by adjusting the availability parameter with time. The $A_{\text {aru }}$ model can be based on the $A_{g r u}$ model with high values of speed and heading-rate to model a fast aircraft.

\section{RESULTS}

Latin hypersquares sampling was used to generate 3000 different scenarios with 9 initialization parameters considered including (1) the number of fire ignition points $n_{\text {fire }} \in[1,7]$; (2) the number of GRU $n_{G R U} \in$ $[1,7]$; (3) a radius for a fire centroid location $r_{\text {cent fire }} \in[0,20]$ from a DA located at cell coordinates $(0,25)$; (4) a fire dispersion radius assumed normally distributed with mean $r_{\text {disp fire }} \in[0,10]$. Similarly for the GRU starting locations there is (5) $r_{\text {cent } G R U} \in[0,20]$ and (6) $r_{d i s p}{ }_{\text {disU }} \in[0,10]$. (7) the starting distance between fire and GRU $r_{\text {fire }}$ GRU. There is also GRU water tank level (8) $w \in[0,1]$ and (9) water range $r_{\text {water }} \in$ $[5,20]$. Additionally, other parameters for fire threat and agents introduce stochasticity in the simulator. These parameters were initialized using the principle of maximum entropy (Jaynes (1957)).

The simulator was implemented in MATLAB and used to generate data for the 3000 scenarios. Various simulator output metrics can be considered such as the maximum time that a fire was within a certain distance of the DA or GRU, the closest distance that any fire came to the DA, or the amount of time that a GRU spent engaging, fleeing, or moving to engage.

We analyzed how effective the BRT was at keeping the fire threat a certain distance away from the DA by considering continuous performance measures such as distances of closest approach. The data was analysed using Generalized Additive Models (GAM) to allow for non-linear effects (Hastie (2017)). Fig. 7 shows the closest approach distances to the DA as a function of the initial distance between the fire and the DA. The distance units are all in cells. The closest approach distances to DA appear to be useful continuous performance measures on the firefighting effectiveness of the BRT. For example, it can be seen that the BRT was able to keep the fire at least 7 cells away from the DA if the initial distance between the DA and fire was 30 cells or greater. It can also be seen that the fire comes dangerously close to the DA (within 2 cells) if the initial distance from asset to fire is 10 cells or less.

We also considered how effective the BRT was at maintaining safe separation between GRU assets and the fire. Fig. 8 shows the closest approach distances to the GRU as a function of the initial distance between the fire and the DA. It can be seen that a dangerously close separation distance of less than 2 cells occurred if the initial distance was less than 8 cells. The closest approach to the GRU would depend on multiple dependant factors including the fire's spread, the effectiveness and aggressiveness of the $\mathrm{C} 2$ and GRU strategies, the sensing range and effectiveness, the water range and the GRU maneuverability.

\section{CONCLUSION}

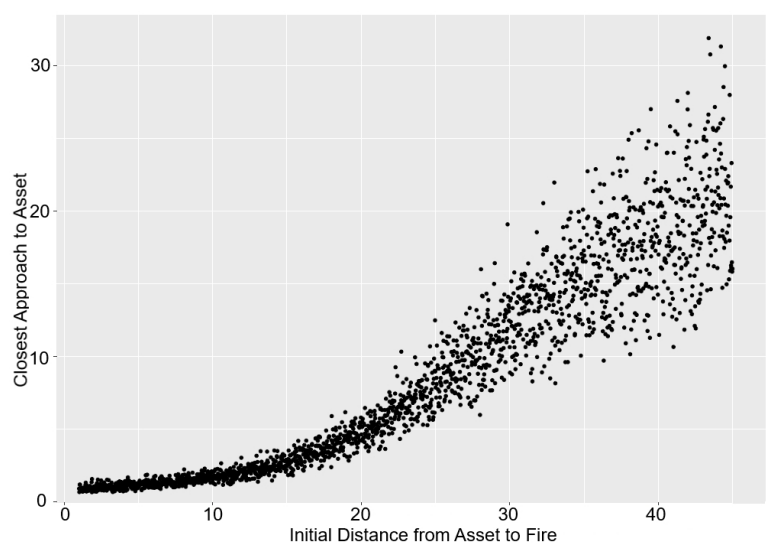

Figure 7. Closest approach distance of fire to the DA as a function of initial distance between the fire and the DA.

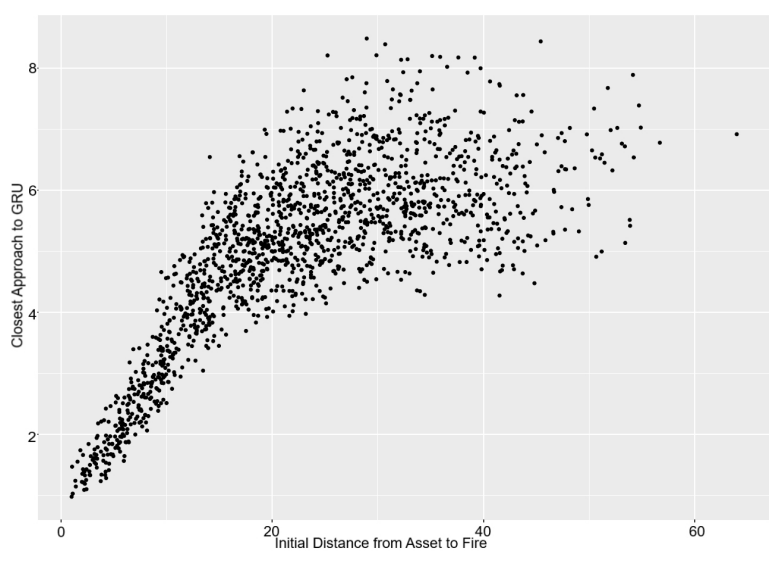

Figure 8. Closest approach distance of fire to the GRU as a function of initial distance between the fire and the DA.

This paper presented a bushfire emergency response simulation tool intended to simulate key dynamic characteristics of emergency response operations. The simulator is intended to be used to assess the impact of uncertainty in complex interacting systems and as a surrogate for similar Defence problems. Future work will consider enhancements for simulation of different sources of uncertainty such as model uncertainties. 


\section{ACKNOWLEDGEMENT}

This research was performed in collaboration with the Defence Science and Technology Group. We thank Mr Wayne Power and Mr Edward Dawson for their helpful advice in the simulator design and facilitation of this research.

\section{REFERENCES}

Alexandridis, A., D. Vakalis, C. I. Siettos, and G. V. Bafas (2008). A cellular automata model for forest fire spread prediction: The case of the wildfire that swept through spetses island in 1990. Applied Mathematics and Computation 204(1), 191-201.

Au, G., S. Goss, C. Heinze, and A. R. Pearce (2000). Rescuemodel: A multi-agent simulation of bushfire disaster management. In Robot Soccer World Cup, pp. 285-290. Springer.

Bruggemann, T., J. J. Ford, G. White, T. Perez, and W. Power (2019). Bushfire emergency response uncertainty quantification. In The 23rd International Congress on Modelling and Simulation (MODSIM2019).

Cunningham, P. and S. J. Delany (2007). k-nearest neighbour classifiers. Multiple Classifier Systems 34(8), 1-17.

Engelmann, H. and F. Fiedrich (2007). Decision support for the members of an emergency operation centre after an earthquake. In Proceedings of the 4th International ISCRAM Conference-Delft, The Netherlands.

Gonzalez, R. A. (2009). Crisis response simulation combining discrete-event and agent-based modeling. In ISCRAM 2009: Proceedings of the 6th International Conference on Information Systems for Crisis Response and Management, Gothenborg, Sweden, 10-13 May 2009. ISCRAM.

Hastie, T. J. (2017). Generalized additive models. In Statistical models in S, pp. 249-307. Routledge.

Hawe, G. I., G. Coates, D. T. Wilson, and R. S. Crouch (2012). Agent-based simulation for large-scale emergency response: A survey of usage and implementation. ACM Computing Surveys (CSUR) 45(1), 8.

Hilton, J., A. L. Sullivan, W. Swedosh, J. Sharples, and C. Thomas (2018). Incorporating convective feedback in wildfire simulations using pyrogenic potential. Environmental modelling \& software 107, 12-24.

HoseinDoost, S., T. Adamzadeh, B. Zamani, and A. Fatemi (2019). A model-driven framework for developing multi-agent systems in emergency response environments. Software \& Systems Modeling 18(3), 1985-2012.

$\mathrm{Hu}, \mathrm{X}$., Y. Sun, and L. Ntaimo (2012). Devs-fire: design and application of formal discrete event wildfire spread and suppression models. Simulation 88(3), 259-279.

Intini, P., E. Ronchi, S. Gwynne, and A. Pel (2019). Traffic modeling for wildland-urban interface fire evacuation. Journal of Transportation Engineering, Part A: Systems 145(3), 04019002.

Jaber, A., F. Guarnieri, and J.-L. Wybo (2001). Intelligent software agents for forest fire prevention and fighting. Safety Science 39(1-2), 3-17.

Jaynes, E. T. (1957). Information theory and statistical mechanics. Physical review 106(4), 620.

Johnston, P., J. Kelso, and G. J. Milne (2008). Efficient simulation of wildfire spread on an irregular grid. International Journal of Wildland Fire 17(5), 614-627.

LaValle, S. M. (2006). Planning algorithms. Cambridge university press.

Liu, Y., H. Liu, Y. Zhou, and C. Sun (2018). Spread vector induced cellular automata model for real-time crown fire behavior simulation. Environmental modelling \& software 108, 14-39.

Massaguer, D., V. Balasubramanian, S. Mehrotra, and N. Venkatasubramanian (2006). Multi-agent simulation of disaster response. In Proceedings of the First International Workshop on Agent Technology for Disaster Management, pp. 124-130.

Miller, C., J. Hilton, A. Sullivan, and M. Prakash (2015). Spark-a bushfire spread prediction tool. In International Symposium on Environmental Software Systems, pp. 262-271. Springer.

Omodei, M. M. and A. J. Wearing (1995). The fire chief microworld generating program: An illustration of computersimulated microworlds as an experimental paradigm for studying complex decision-making behavior. Behavior Research Methods, Instruments, \& Computers 27(3), 303-316.

Padgham, L., K. Nagel, D. Singh, and Q. Chen (2014). Integrating BDI agents into a MATSim simulation.

Shahparvari, S., P. Chhetri, B. Abbasi, and A. Abareshi (2016). Enhancing emergency evacuation response of late evacuees: Revisiting the case of australian black saturday bushfire. Transportation research part E: logistics and transportation review 93, 148-176.

Singh, D. and L. Padgham (2017). Emergency evacuation simulator (ees)-a tool for planning community evacuations in australia. In IJCAI, pp. 5249-5251.

Sullivan, A. L. (2009). Wildland surface fire spread modelling, 1990-2007. 3: Simulation and mathematical analogue models. International Journal of Wildland Fire 18(4), 387-403.

Tolhurst, K., B. Shields, D. Chong, et al. (2008). Phoenix: development and application of a bushfire risk management tool. Australian Journal of Emergency Management, The 23(4), 47.

Wooldridge, M. (2009). An introduction to multiagent systems. John Wiley \& Sons.

Yongzhong, Z., E. Youhao, H. Tao, Z. Songbing, and W. Jihe (2005). A ca-based information system for surface fire spreading simulation. In Proceedings. 2005 IEEE International Geoscience and Remote Sensing Symposium, 2005. IGARSS'05., Volume 5, pp. 3484-3487. IEEE. 